\title{
Aortoplastia trapezoidal: proposta técnica para correção cirúrgica da coarctação de aorta
}

\author{
Jarbas J. DINKHUYSEN*, Luiz Carlos Bento de SOUZA*, Paulo CHACCUR* Antoninho S. ARNONI**,
} Camiilo ABDULMASSIH NETO*, Walmir F. FONTES ${ }^{\star}$, Paulo P. PAULISTA ${ }^{\star *}$, Adib D. JATENE ${ }^{\star \star \star}$

RBCCV 44205-249

DINKHUYSEN, J. J.; SOUZA, L. C. B.; CHACCUR, P.; ARNONI, A. S.; ABDULMASSIH NETO, C.; FONTES, V. F.; PAULISTA, P.P.; JATENE, A. D. - Aortoplastia trapezoidal: proposta para correção cirúrgica da coarctaçăo de aorta. Rev. Bras. Cir. Cardiovasc., 9 (4): 205-212, 1994.

RESUMO: O cirurgião muitas vezes encontra dificuldade em recompor o diâmetro interno quando realiza a anastomose término-terminal no tratamento cirúrgico da coarctação da aorta. Um dos fatores determinantes desta dificuldade são as alterações anatômicas típicas da região coarctada, cujo discernimento cirúrgico nem sempre é simples, a despeito de inúmeras e consagradas opçōes técnicas disponíveis. Nas crianças este fato pode se revestir de importância, dada a eventual possibilidade do reaparecimento de gradientes pressóricos, sendo até possível uma nova intervenção cirúrgica, em alguns casos. Diante disto, os autores apresentam uma proposta técnica cujo objetivo é otimizar o diâmetro da aorta ao nível da anastomose, minimizando, assim, a possibilidade do aparecimento de possíveis gradientes a longo prazo. Assim sendo, após a remoçāo da área coarctada, são criados, em cada coto, três retalhos de aspecto trapezoidal, os quais, ao serem aproximados, se encaixam à semelhança de uma engrenagem. Estes retalhos trapezoidais são obtidos pela ressecçāo de três cunhas idênticas e eqũidistantes em cada coto e a anastomose, com pontos contínuos ou separados, é feita de modo a encaixar as saliências trapezoidais de um coto nas reentrâncias intertrapezoidais do outro coto, dando como resultado uma linha de sutura de aspecto sinusoidal, em zig-zag. Até o presente, foram operados por esta técnica 4 pacientes, cujas idades foram: 2 meses, 4 meses, 10 anos e 36 anos (masculinos, 3). Apenas o primeiro caso tinha cardiopatia congênita associada (OAVC parcial) e todos tinham CoAo severa e HAS. Năo ocorreram complicaçōes operatórias, tendo-se observado boa evoluçăo, até o momento. O primeiro caso ( 2 meses com cardiopatia congênita) recoarctou no $9^{\circ}$ mês de evolução, tendo sido necessária reintervenção, devido, provavelmente, a problemas de técnicas ou indicação inapropriada. 0 método proposto permite restituir e até mesmo aumentar o diâmetro interno da aorta na área de sutura, dependendo, para tanto, do grau de profundidade dos retalhos trapezoidais de cada coto.

DESCRITORES: aorta, coarctação, cirurgia.

\section{INTRODUÇÃO}

Já está completando meio século desde a primeira publicação ${ }^{4}$ do tratamento cirúrgico com sucesso da coarctação de aorta (CoAo) pela técnica de ressecção da área coarctada seguida de anastomose término-terminal e ainda persistem fortes controvérsias quanto ao manuseio desta anomalia congênita, principalmente no recém-nascido.

A variedade do quadro anatômico, as associações com defeitos congênitos intracardíacos, a exteriorização clínica precoce ou tardia, além de

\footnotetext{
Trabalho realizado no Instituto Dante Pazzanese de Cardiologia e no Hospital do Coração da Associação do Sanatório Sírio. São Paulo, SP, Brasil. Apresentado ao $21^{\circ}$ Congresso Nacional de Cirurgia Cardiaca. Porto Alegre, RS, 24 a 26 de março, 1994.* Do Instituto Dante Pazzanese de Cardiologia e do Hospital do Coração da Associação do Sanatório Sírio.

** Do Instituto Dante Pazzanese de Cardiologia.

*** Do Hospital do Coração da Associação do Sanatório Sírio.

Endereço para correspondência: Jarbas Dinkhuysen. Rua Desembargador Eliseu Guilherme, 123. Centro Cirúrgico. 04004-030 São Paulo, SP, Brasil.
} 
DINKHUYSEN, J. J.; SOUZA, L. C. B.; CHACCUR, P.; ARNONI, A. S.; ABDULMASSIH NETO, C.; FONTES, V. F.; PAULISTA, P P.; JATENE, A. D. - Aortoplastia trapezoidal: proposta para correção cirúrgica da coarctação de aorta. Rev. Bras. Cir. Cardiovasc., 9 (4): 205-212, 1994.

outros fatores, compõem o amplo espectro desta peculiar malformação, cuja manipulação clínico-cirúrgica nem sempre é simples.

Genericamente, existem cinco técnicas bem delimitadas, a saber: a ressecção da área coarctada seguida da anastomose término-terminal ${ }^{4}$; a ampliação do local da estenose por meio de um retalho de tecido biológico, heterólogo ou artificial ${ }^{17}$; a ampliação do local da CoAo com o tecido da artéria subclávia do paciente, descrita por WALDHAUSEN \& NARWOLD 18; a ampliação da área coarctada através do reimplante da artéria subclávia, técnica proposta por MENDONÇA et alii ${ }^{13} \mathrm{e}$, finalmente, a ressecção da área coarctada seguida da interposição de um tubo de tecido biológico heterólogo ou artificial ${ }^{2}$. Vários autores $2,5,11$ publicaram variações sobre estes métodos, quase sempre relacionados com alterações anatômicas incomuns encontradas na aorta.

No presente trabalho os autores apresentam proposta técnica para a correção cirúrgica da coarctação da aorta. Trata-se da aortoplastia trapezoidal como variação técnica da anastomose término-terminal, cujo objetivo é aumentar o diâmetro da aorta ao nível da sutura, minimizando, assim, o surgimento de possíveis gradientes a longo prazo.

\section{CASUÍSTICA, MÉTODOS E RESULTADOS}

O método proposto se apoia em elementos de geometria; há a confrontação linear hipotética de trapezóides (Figura 1) de dois retângulos planos, de maneira a encaixar os vértices dos trapezóides de um lado nas reentrâncias intertrapezoidais, do outro à semelhança de uma engrenagem, o que neces-

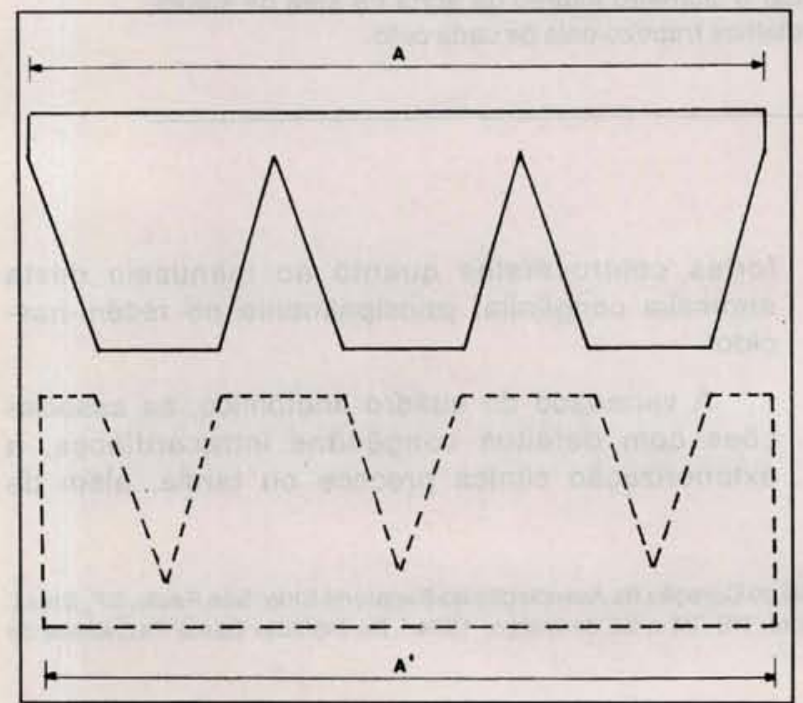

Fig. 1- Dois retângulos planos com três trapezóides de cada lado confrontados. sariamente adiciona um segmento extra no local da confrontação, dando como resultado final uma distância maior do que as bases dos retângulos.

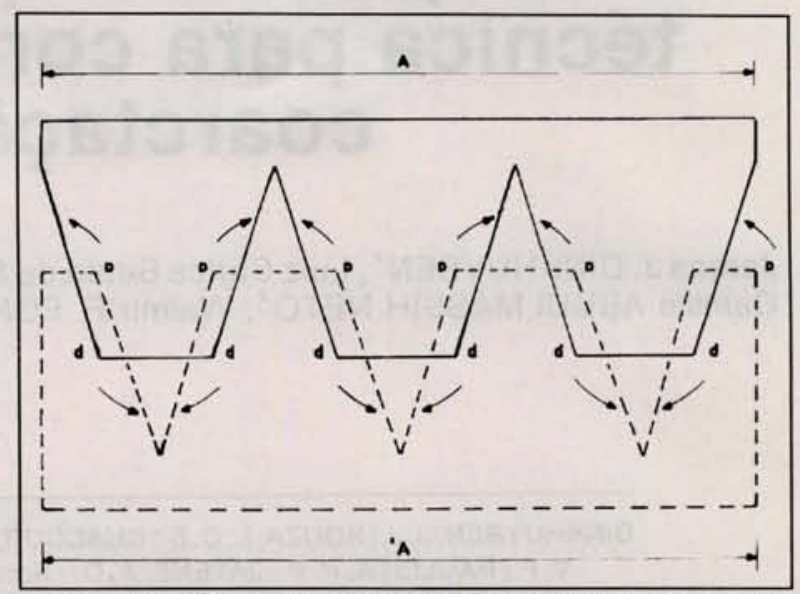

Fig. 2 - Dois retângulos planos com três trapezóides de cada lado, sendo hipoteticamente encaixados, à semelhança de uma engrenagem.

No momento em que aproximarmos os retângulos, interfaciando os trapezóides e admitindo-se hipoteticamente um encaixe elástico perfeito, a distância resultante deste encaixe (B) necessariamente será maior que a original do retângulo A (Figuras $1,2$ e 3$)$.

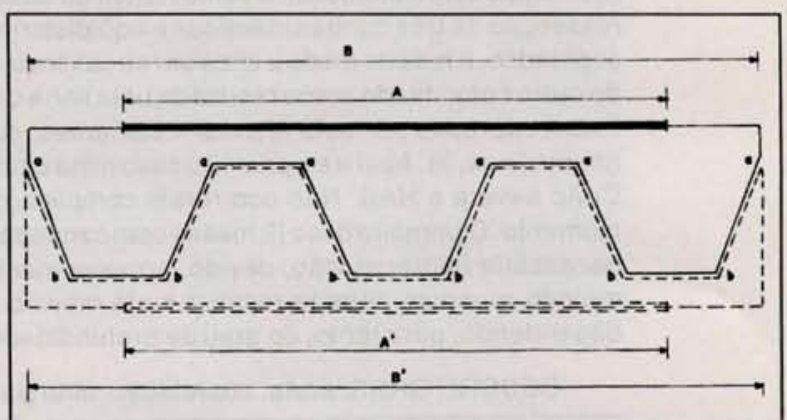

Fig. 3 - Dois retângulos figuras planas com três trapezóides hipoteticamente já encaixados, notando-se significativo aumento na distância na linha de confrontação final $\left(A=A^{\prime}\right)<B$.

Se levarmos este raciocínio para um tubo (aorta) e, conforme a Figura 4, aplicarmos dados matemáticos, concluiremos que o vértice de cada trapezóide é igual a $2 \mathrm{Pi} \times \mathrm{r} / 3(\mathrm{r}=$ raio $)$.

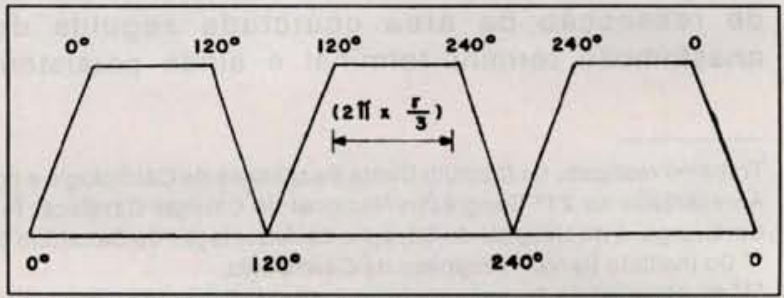

Fig. 4 - Figura plana com três trapezóides representando um tubo (aorta) extendido. 
DINKHUYSEN, J. J.; SOUZA, L. C. B.; CHACCUR, P.; ARNONI, A. S.; ABDULMASSIH NETO, C.; FONTES, V. F.; PAULISTA, P. P.; JATENE, A. D. - Aortoplastia trapezoidal: proposta para correção cirúrgica da coarctação de aorta. Rev. Bras. Cir. Cardiovasc., 9 (4): 205-212, 1994.

Logo, o perímetro $(P)$ de cada trapezóide é igual

$P=2 \cdot n \cdot L+P i \cdot d$

$\mathrm{n}=$ número de reentrância intertrapezoidal

$\mathrm{L}=$ profundidade das reentrâncias intertrapezoidais

d = diâmetro inicial do tubo (aorta)

Para o cálculo do perímetro final após o confronto de dois cotos tubulares (aorta) com três trapezóides de cada lado, conforme já foi descrito, fica:

$$
P=6 . L+P i . d
$$

Como $L$ (profundidade das reentrâncias intertrapezoidais) é a única variável, o acréscimo sobre o perímetro inicial é diretamente proporcional à profundidade destas reentrâncias intertrapezoidais.

Transpondo este princípio ao manuseio de tecidos biológicos cuja capacidade de adaptação espacial às mais variadas formas é plena, a Figura 5 mostra o resultado final após aproximarmos e confrontarmos os vértices de um lado $\mathrm{com}$ as reentrâncias intertrapezoidais $e$, do outro, observando-se adaptação às formas geométricas, dando como resultado final significativo aumento de distância $\left(A=A^{\prime}\right)<\left(B=B^{\prime}\right)$

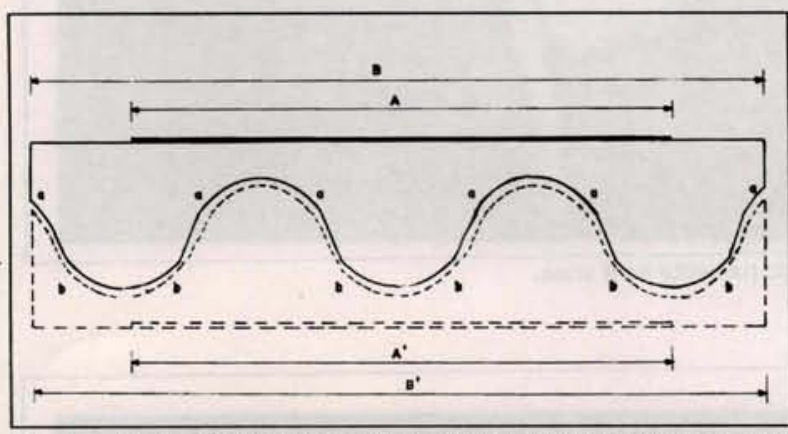

Fig. 5 - Resultado final da confrontação de tecidos elásticos com estas formas trapezoidais $\left(A=A^{\prime}\right)<\left(B=B^{\prime}\right)$.

A finalidade de detalhar os princípios sobre os quais se alicerça a técnica de aortoplastia trapezoidal para a correção cirúrgica da coarctação da aorta foi a de tornar mais compreensivel a aplicação de princípios de geometria em medicina, mais particularmente em técnica cirúrgica.

Os esquemas da Figura 6 detalham a técnica proposta. Após a dissecção é isolada a região coarctada e adjacências, é feito o pinçamento da aorta proximal e distal e após a secção do ligamento arterioso ou secção e sutura do canal arterial, caso presente, a área coarctada, juntamente com o tecido justaductal, são ressecados. A seguir, são criados em cada coto três retalhos de aspecto trapezoidal, que são obtidos pela ressecção de três cunhas idênticas e eqüidistantes. A anastomose é iniciada pela aproximação do vértice de um trapezóide de um coto na reentrância intertrapezoidal do outro coto, à semelhança de uma engrenagem. A anastomose com pontos contínuos ou separados é iniciada, resultando, ao final, uma linha de sutura sinusoidal (zig-zag), aumentando sobremaneira o diâmetro da aorta a este nível.

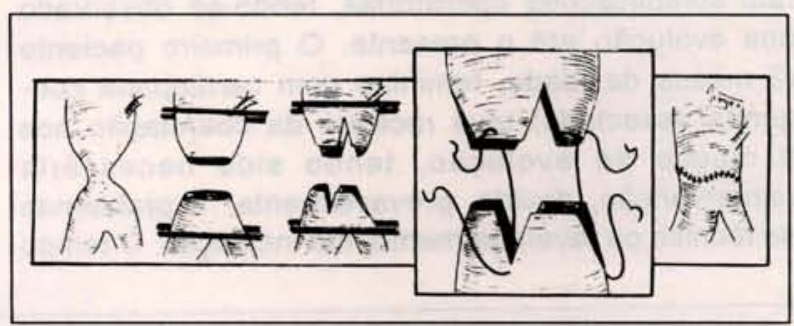

Fig. 6 - Esquema mostrando os tempos cirúrgicos da técnica da anastomose trapezoidal na correção cirúrgica da coarctação da aorta.

É necessário salientar que, ao se realizar anastomose de um vaso com cotos idênticos ao seu diâmetro original, de acordo com os princípios aqui descritos, o resultado final é um acréscimo de diâmetro na região da linha de sutura (Figura 7).

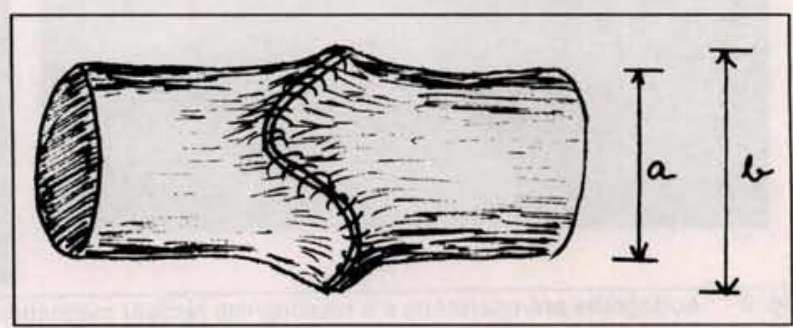

Fig. 7 - Anastomose trapezoidal de um vaso com cotos idênticos ao diâmetro original $(\mathrm{a}<\mathrm{b})$.

Já com cotos de diâmetros menores que o diâmetro original do vaso, após concluída a anastomose observa-se a recomposição do diâmetro original do vaso (Figura 8 ).

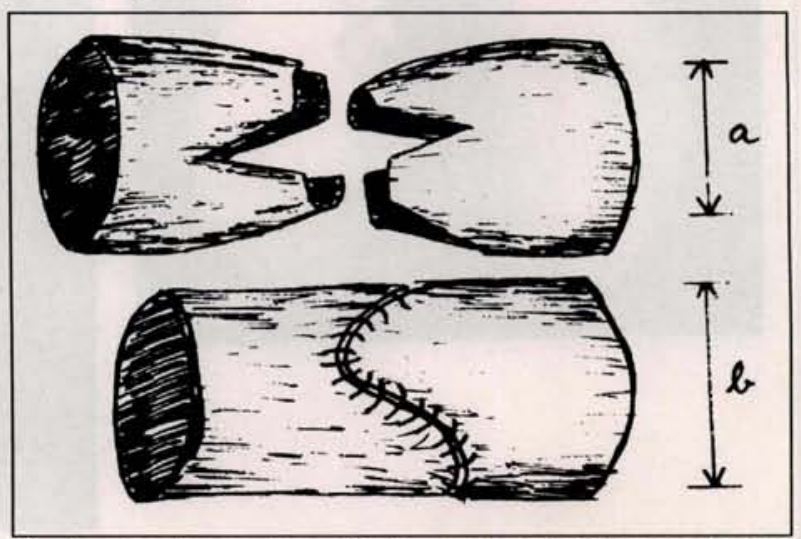

Fig. 8 - Anastomose trapezoidal num vaso com cotos menores que 0 diâmetro original do vaso $(\mathrm{a}=\mathrm{b})$. 
DINKHUYSEN, J. J.; SOUZA, L. C. B.; CHACCUR, P.; ARNONI, A. S.; ABDULMASSIH NETO, C.; FONTES, V. F.; PAULISTA, P. P.; JATENE, A. D. - Aortoplastia trapezoidal: proposta para correção cirúrgica da coarctação de aorta. Rev. Bras. Cir. Cardiovasc., 9 (4): 205-212, 1994.

Foram operados por esta técnica 5 pacientes com idades de 2 meses ( 2 casos), 7 meses, 8 anos e 36 anos, sendo 3 do sexo masculino. Dois pacientes tinham cardiopatia congênita associada: defeito do septo atrioventricular forma parcial (OSVC) e persistência do canal arterial (PCA), respectivamente, e todos eram portadores de CoAo severa e hipertensão arterial sistêmica (HAS). Não ocorreram complicações operatórias, tendo-se observado boa evolução até o presente. O primeiro paciente (2 meses de idade, feminino com cardiopatia congênita associada) teve recidiva da coarctação aos 9 meses de evolução, tendo sido necessária reintervenção, devido, provavelmente, a problemas de técnica ou, eventualmente, má indicação. O tempo de evolução pós-operatória em todos os casos é inferior a um ano.

Dois pacientes foram reestudados, podendo se comparar os dados angiográficos pré e pós-operatórios. As Figuras 9 e 10 mostram a aortografia préoperatória, onde se observam coarctação da aorta praticamente cega e a ressonância nuclear magnética feita na $3^{\mathrm{a}}$ semana de $\mathrm{PO}$ demonstrando a normalização do diâmetro da aorta ao nível da anastomose, no paciente de 8 anos.

A Figura 10 mostra a aortografia pré-operatória onde se observa coarctação da aorta e a ressonância nuclear magnética, feita no $11^{\circ}$ mês de $\mathrm{PO}$, evidenciando a normalização da luz da aorta, no paciente de 30 anos.

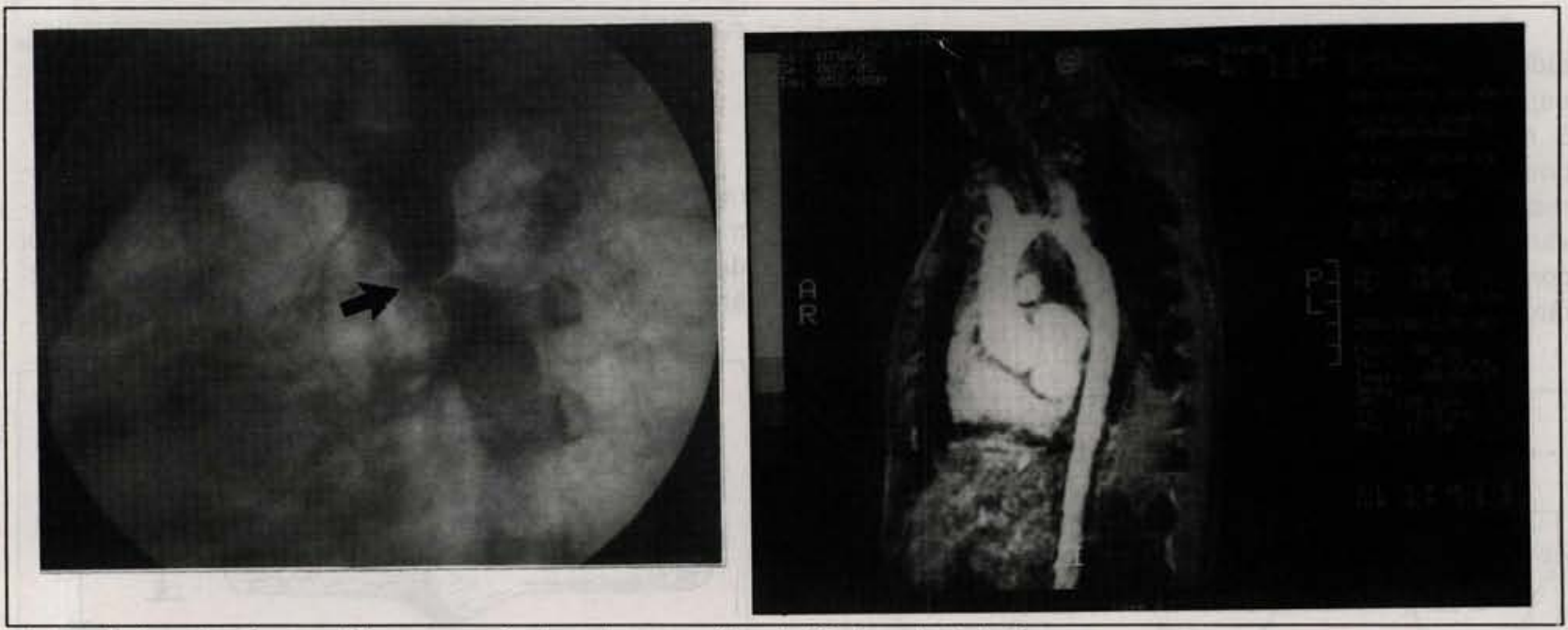

Fig. 9 - Aortografia pré-operatória e a ressonância nuclear magnética do PO do paciente de 8 anos.

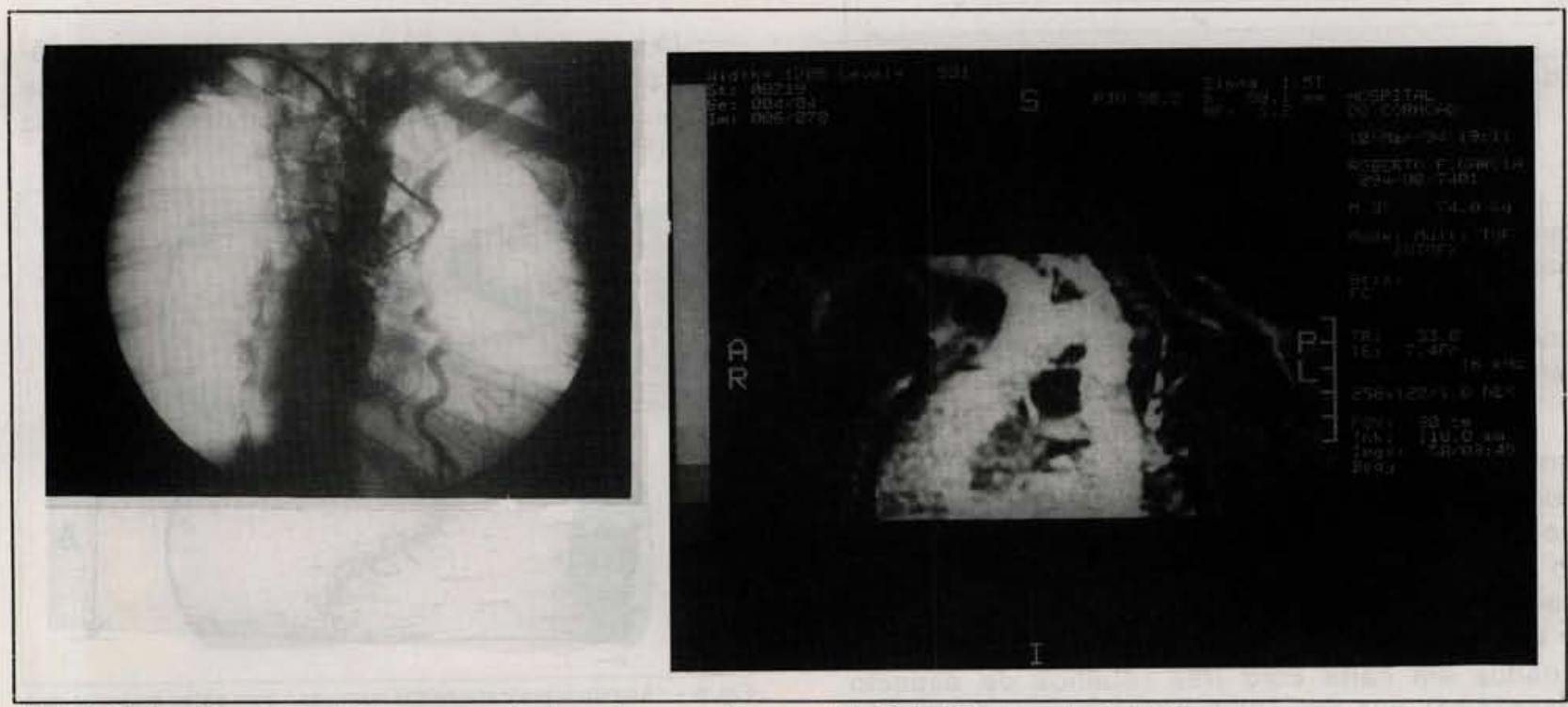

Fig. 10 - Aortografia pré-operatória e a ressonância nuclear magnética, no $11^{\circ}$ mês de $\mathrm{PO}$, do paciente de 36 anos. 
DINKHUYSEN, J. J.; SOUZA, L. C. B.; CHACCUR, P.; ARNONI, A. S.; ABDULMASSIH NETO, C.; FONTES, V. F.; PAULISTA, P. P.; JATENE, A. D. - Aortoplastia trapezoidal: proposta para correção cirúrgica da coarctação de aorta. Rev. Bras. Cir. Cardiovasc., 9 (4): 205-212, 1994.

\section{COMENTÁRIOS}

A técnica de ressecção da CoAo seguida de anastomose término-terminal descrita por CRAFOORD \& NYLIN ${ }^{4}$ é o único método que remove o tecido da área coarctada com o tecido justaductal, que é, do ponto de vista histológico, potencialmente patológico. Para se obter uma anastomose término-terminal ampla sem grandes traçōes, é necessário generosa dissecção da porção distal do arco aórtico, artéria subclávia esquerda e da aorta torácica. Esta manipulação cirúrgica pode envolver algum risco ao nervo vago, ao nervo laríngeo recorrente, ao ducto torácico e, eventualmente, às artérias intercostais, geralmente calibrosas. A despeito dos cuidados, a feitura de anastomose ampla que restitua o diâmetro normal da aorta nem sempre é conseguida, sendo necessário ampliar a anastomose para evitar gradiente pressórico residual. Este aspecto é particularmente importante quando o paciente é recém-nascido.

O caso de um paciente de 30 anos ilustra bem estas considerações. A Figura 11 mostra a aortografia da porção ascendente e do joelho posterior da aorta, de paciente portador de CoAo, tratado com anastomose término-terminal aos 8 anos de idade e no momento apresenta aneurisma da porção ascendente da aorta e uma estenose (gradiente 30 $\mathrm{mmHg}$ ) ao nível da anastomose. Vinte e dois anos após, é possível especular que esta reestenose possa ter influenciado a formação do aneurisma.

Ampliação da região coarctada com um retalho de tecido biológico ${ }^{17}$, Dacron ou PTFE, requer o mínimo de dissecção regional e as estenoses ístmícas ou tubulares, geralmente de difícil resolução, não constituem maior dificuldade, pois o reta-
Iho pode ser extendido tanto para a porção proximal e/ou distal da aorta torácica. A questão de ressecção ou não do tecido coarctado é controverso e alguns autores ${ }^{8}$ não recomendam, tendo em vista $\circ$ enfraquecimento da parede da aorta neste local, com possibilidades de formações aneurismáticas a longo prazo. Em contraposição, a permanência deste tecido na luz da aorta pode ser fator de reestenose a longo prazo. Tanto o ligamento arterioso quanto o canal arterial requerem a secção respectiva, liberando completamente a aorta, evitando, assim, eventuais retrações, principalmente ao nível da sutura.

WALDHAUSEN \& NAHRWOLD ${ }^{18}$ descreveram a aortoplastia (amplificação com a artéria subclávia constituindo uma opção técnica de excelência a ser aplicada, principalmente nos pacientes pediátricos. Além de requerer pequena dissecção regional e preservação das artérias intercostais, a ampliação da estenose é feita através de tecido da artéria subclávia esquerda, com possibilidades de crescimento. A maior desvantagem deste método é a ligadura da artéria subclávia e os ramos distais, especialmente a artéria vertebral, que deve ser ligada para prevenir mecanismo tardio de roubo de fluxo. Ao preservar a artéria torácica interna e o tronco tireocervical, é possível que se promova aumento de circulação colateral ao braço esquerdo, mas, em contrapartida, diminui o tamanho do retaIho de subclávia a ser suturado sobre a região coarctada; uma ampliação satisfatória exige que o retalho ultrapasse pelo menos $12 \mathrm{~mm}$ a região estenótica. Em razão disto, nas coarctações ístmicas muitas vezes o método não pode ser aplicado; a aortotomia muito longa poderá exigir tração exagerada no retalho, o que constitui fator predisponente de estenose a longo prazo.

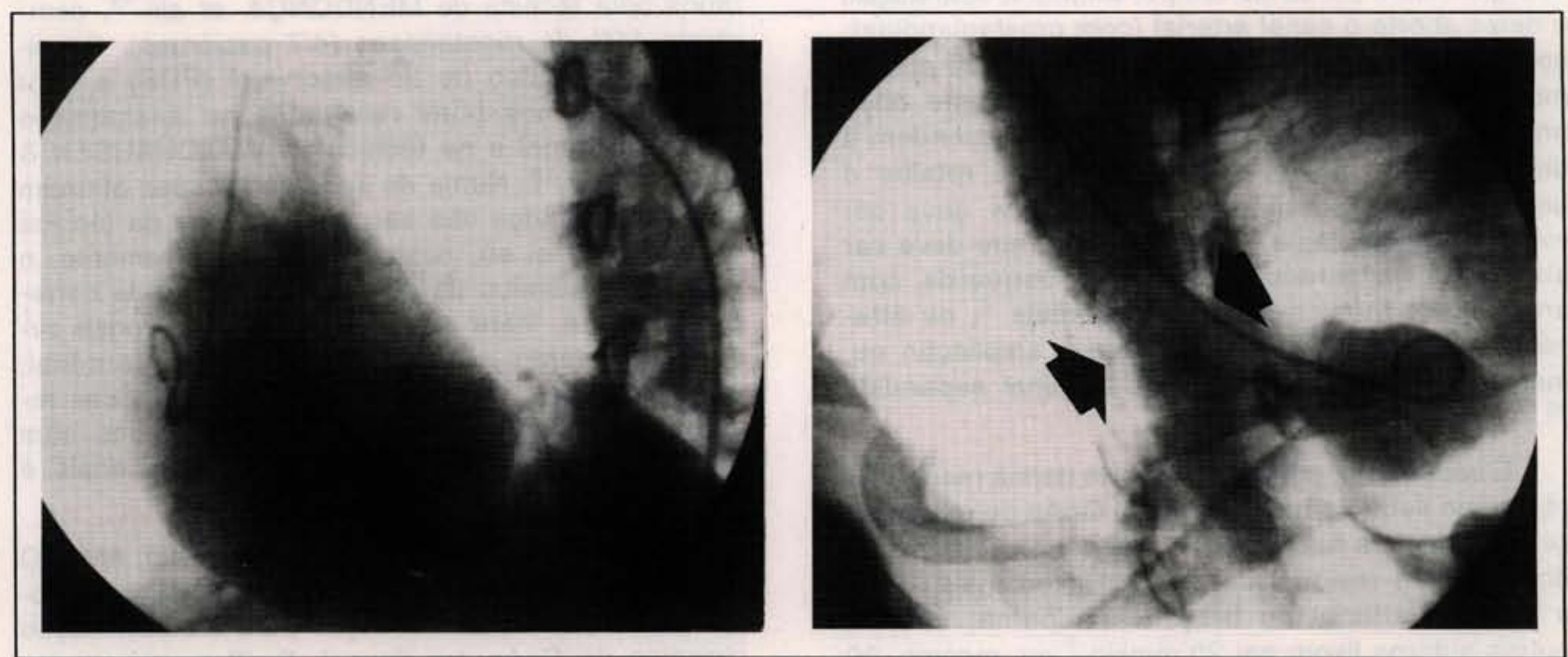

Fig. 11 - Aortografia da porçăo ascendente da aorta com aneurisma e uma reestenose ( $30 \mathrm{~mm} \mathrm{Hg}$ gradiente) no local da sutura. 
DINKHUYSEN, J. J.; SOUZA, L. C. B.; CHACCUR, P.; ARNONI, A. S.; ABDULMASSIH NETO, C.; FONTES, V. F.; PAULISTA, P. P.; JATENE, A. D. - Aortoplastia trapezoidal: proposta para correção cirúrgica da coarctação de aorta. Rev. Bras. Cir. Cardiovasc., $9(4): 205-212,1994$.

A aortoplastia por reimplante da artéria subclávia sobre a região coarctada, descrita em nosso meio por MENDONÇA et alii ${ }^{13}$, tenta incorporar as vantagens da aortoplastia com retalho arterial (técnica de Waldhausen) eliminando a ligadura da artéria subclávia. É necessário, contudo, observar algns aspectos técnicos, principalmente na ampla dissecção das porções distais da artéria subclávia esquerda com possível ligadura e secção da artéria vertebral e torácica interna, para a boa mobilização do vaso e permitir uma anastomose sem tensão. A técnica seria melhor aplicável em crianças maiores e nas coarctações localizadas e mais proximais, embora o reimplante, com suficiente ampliação da área coarctada fique sem tensão, tanto no vaso como na linha de sutura.

Em relação ao uso de "retalho" para ampliação, UNGERLEIDER ${ }^{15}$, em apoio aos críticos do método, acredita que a técnica poderia provocar, tardiamente, o aparecimento de aneurismas. Todavia, os seus resultados são bastante consistentes com a mortalidade $(0 / 54)$ e a recoarctação $(2 / 54)$, provavelmente por problemas técnicos; não resseca o tecido da coarctação, pois pode provocar o aparecimento de aneurismas, e com retalho amplo, procura eliminar qualquer possibilidade anatômica do aparecimento de gradientes residuais. As vantagens são de uma técnica simples e efetiva, com mínimo de manipulação cirúrgica. A ampliação com retalho de tecido deve ser considerada sob certas circunstâncias nos casos de CoAo associada a cardiopatia congênita, quando os dois defeitos são tratados num tempo único por toracotomia mediana. Outra indicação são os casos de emergência, os recémnascidos com CoAo crítica e ventrículo esquerdo de dimensões reduzidas $(20 \mathrm{ml}-25 \mathrm{ml} / \mathrm{m} 2)$, nos quais o cirurgião, sem perda de tempo, amplia a coarctação e deixa aberto o canal arterial (com prostagiandina), como via de escape em alívio à hipertensão pulmonar. Nos casos de recoarctação recurrente após anastomose término-terminal que não respondem à dilatação com balão, a ampliação com retalho é uma boa opção cirúrgica. Esta técnica deve ser evitada na hipoplasia do arco aórtico, que deve ser abordado via toracotomia lateral esquerda com anastomose término-terminal expandida ${ }^{6}$, ou através de toracotomia mediana com a ampliação ou, mesmo, a anastomose término-terminal expandida 16.

É necessário, entretanto, que se defina recoarctação como sendo a recorrência da CoAo ou reestenose, para alguns autores ${ }^{19}$ como sendo a presença de gradiente residual de $10 \mathrm{mmHg}$ ou mais, entre a pressão arterial no braço e na perna; embora outros autores fixem em $20 \mathrm{mmHg}^{7}$ ou, mesmo, 30 $\mathrm{mmHg}$ esta diferença.
JONAS ${ }^{9}$ afirma a evidência histológica na coarctação justaductal de tecido muscular fino de origem ductal que se fibrosa posteriormente, podendo ser o local de uma dilatação aneurismática; o fato poderá constituir uma desvantagem para as técnicas que não removem este tecido e, assim, o autor considera que a ressecção da área coarctada seguida da anastomose término-terminal é a melhor opção.

A despeito do fato de a mortalidade hospitalar ter diminuído, a intervenção precoce deve ser restringida aos portadores de insuficiência cardíaca resistente a tratamento clínico. Em alguns casos um defeito intracardíaco e, mesmo, a presença do canal arterial associados a CoAo são fatores indicativos da operação.

Um dos maiores problemas após a correção da CoAo em recém-nascidos é a reestenose, talvez mais dependente da idade do que da técnica operatória ${ }^{19}$. A ressecção seguida da anastomose término-terminal tem sido freqüentemente responsável por indices importantes de reestenose 19. KORFER et alii ${ }^{10}$ relataram indices de reestenose em $9 \%$ dos seus pacientes, afirmando que isto se deve mais a problemas técnicos do que, propriamente, às condições da anatomia da aorta.

Em contraposição, MESSMER et alii ${ }^{14}$ confirmam os bons resultados obtidos com a anastomose término-terminal ( $1 / 11$ casos com idade acima de 1 mês de vida), assim como pela técnica de Waldhausen $(0 / 17)$ com pacientes abaixo e acima de 1 mês de vida. Já a ampliação com retalho de tecido, os resultados foram mais comprometidos, sendo que $50 \%$ (crianças abaixo de 1 ano) e $25 \%$ (acima de 1 ano de vida) apresentaram reestenose. Relatam que, apesar do reduzido número de pacientes operados pela técnica de MENDONÇA et alii ${ }^{13}$, ocorreram $44 \%$ de reestenoses ( $4 / 7$ pacientes). Consideram que o uso de fio absorvivel (PDS) é fator fundamental nos bons resultados na anastomose término-terminal e na técnica de WALDHAUSEN \& NAHRWOLD ${ }^{18}$. Numa de suas conclusōes afirmam que os resultados não são dependentes da técnica cirúrgica por si só, concorrendo, decisivamente, o aspecto anatômico da aorta no momento da correção cirúrgica. Para coarctações com estenose circunscrita, tanto a anastomose término-terminal, como a técnica de Waldhausen apresenta bons resultados. A indicação para as coarctações com estenoses tubulares e malformações adicionais é para ampliação com retalho de tecido.

Numa revisão apurada sobre o assunto, AMATO et alii ${ }^{1}$ abordam o problema com elegância e propriedade, chamando a atenção para a classificação cirúrgica de CoAo em tipos I, II, III e preconizam para os recém-nascidos a anastomose término-ter- 
DINKHUYSEN, J. J.; SOUZA, L. C. B.; CHACCUR, P.; ARNONI, A. S.; ABDULMASSIH NETO, C;; FONTES, V. F.; PAULISTA, P. P.; JATENE, A. D. - Aortoplastia trapezoidal: proposta para correção cirúrgica da coarctação de aorta. Rev. Bras. Cir. Cardiovasc., 9 (4): 205-212, 1994.

minal, a de Waldhausen para a CoAo localizada, procurando evitar a ampliação com retalho de tecido, mesmo que seja de própria artéria subclávia, dada a possibilidade de retratação fibrosa e eventual recoarctação. Para o tipo II, isto é, CoAo com hipoplasia ístmica, tanto a técnica de Waldhausen, como a anastomose término-terminal e o reimplante da artéria subclávia ${ }^{12}$ ou, eventualmente, a anastomose término-terminal expandida 6, 11 podem ser aplicadas. No caso da ampliação com retalho de tecido, não recomendam a ressecção do tecido interno da CoAo, devido à fraqueza da parede do vaso, ensejando a possibilidade de formação aneurismática a longo prazo ${ }^{1}$. No tipo III, isto é, CoAo com hipoplasia envolvendo o ístmo da aorta e os segmentos entre a carótida e a subclávia esquerda, a aortoplastia expandida, conforme advogam ELLIOT ${ }^{6}$ e LACOUR-GAYET et alii ${ }^{11}$, é a mais indicada. A conclusão é que não existe uma técnica de aplicação indiscriminada, sendo as condições anatomopatológicas da aorta que orientarão o tipo de correção.

A aortoplastia trapezoidal, proposta pelos auto- res como opção ao tratamento cirúrgico da CoAo, encontra espaço nesta variàda găma de técnicas. Suas vantagens dizem respeito à importante otimização do diâmetro interno da aorta no local da anastomose. Assim como para a anastomose término-terminal, exige ampla dissecção dos segmentos arteriais envolvidos; remove a área coarctada, eliminando a presença do tecido justaductal e do esporão interno da CoAo potencialmente degeneráveis. Não é um método de aplicação indiscriminada, pois, nos casos de hipoplasia tubular, com ou sem envolvimento do arco aórtico, a melhor indicação é a aortoplastia expandida, com ou sem reimplante de artéria subclávia. Do ponto de vista teórico, desde que executada com rigor técnico, a aortoplastia trapezoidal proporciona o aumento da luz arterial, evitando a possibilidade de reestenose. A disposição sinusoidal e não retilínea da sutura pode constituir um fator impeditivo de retração fibrosa linear, à medida em que o diâmetro da aorta aumente proporcionalmente ao desenvolvimento da criança. A forma sinusoidal da sutura pode acompanhar o aumento do diâmetro do vaso, evitando, assim, a diminuição da luz arterial.

RBCCV 44205-249

DINKHUYSEN, J. J.; SOUZA, L. C. B.; CHACCUR, P.; ARNONI, A. S.; ABDULMASSIH NETO, C.; FONTES,

V.F.; PAULISTA, P.P.; JATENE, A. D. - Trapezoidal aortoplasty: technic proposal for aortic coarctation. Rev. Bras. Cir. Cardiovasc., 9 (4): 205-212, 1994.

ABSTRACT: The authors propose a technical variation that aimes to optimize the aorta diamenter at the site of the anastomosis, obviating the chance of developing fibrosis retraction and intraortic gradients at the long term follow-up. Following the removal of the coartation site, 3 trapezoidal flaps are shaped at each aorta end. These flaps when approached will fit perfectly. These flaps are shaped by 3 identical coinning at each aortic end, and the anastomosis will approached the projection of one end to the recess of the other. The result is a zig-zag sinusoid like suture. Until now 5 patients underwent this technique with age 2 months, 4 months, 10 and 36 years. Three were male. There were no surgical complication. The first case ( 2 months with associated congenital cardiopathy) had recoarctation in the 9th month of folow up, and required reintervention. This was problably due to technical difficulties or inadequate indication. The proposed technique yelds a large lumen, maybe even larger than the reference diameter at the anastomosis site. Such diameter depends on the flaps depht in each aortic end.

DESCRIPTORS: aortic coarctation, surgery. 
DINKHUYSEN, J. J.; SOUZA, L. C. B.; CHACCUR, P.; ARNONI, A. S.; ABDULMASSIH NETO, C.; FONTES, V. F.; PAULISTA, P. P.; JATENE, A. D. - Aortoplastia trapezoidal: proposta para correção cirúrgica da coarctação de aorta. Rev. Bras. Cir. Cardiovasc., 9 (4): 205-212, 1994.

\section{REFERÊNCIAS BIBLIOGRÁFICAS}

1 AMATO, J. J.; GALDIERUI, R. J.; COTRONEO, V. Role extended aortoplasty related to the definition of coarctation of the aorta. Ann. Thorac. Surg., 52: 615-620, 1991.

2 AMATO, J. J.; RHEINLANDER, H. F.; CLEVELAND, R. J. - A method of enlarging the distal transverse aorta in infants with hypoplasia and coarctation of the aorta. Ann. Thorac. Surg., 23: 261-262, 1977.

3 BEECKMAN, R. H.; ROCCHINI, A. P.; BEHRENDT, D. M.; ROSENTHAL, A. - Reoperation for coarctation of the aorta. Am. J. Cardiol, 48: 1108-1114, 1981.

4 CRAFOORD, C. \& NYLIN, G. - Congenital coarctation of the aorta and its surgical treatment. J. Thorac. Cardiovasc. Surg., 14: 347-352, 1945.

DIETL, C. A. \& TORRES, A. R. - Coarctation of the aorta: anastomotic enlargement with subclavian artery: two new surgical option. Ann. Thorac. Surg., 43: 224-225, 1987.

6 ELLIOT, M. J. - Coarctation of the aorta with arch hypoplasia: improvements on a new technique. Ann. Thorac. Surg., 44: 321-323, 1987.

7 HARTMANN Jr., A. F.; GOLDRING, D.; HERNANDEZ, A. - Recurrent coarctation of the aorta after successful repair in infuncy. Am. J. Cardiol., 25: 405-410, 1970.

8 HEHRLEIN, F. W.; MULCH, J.; RANTENBURG, H. W.; SCHLEPPER, M.; SHELD, H. H. - Incidence and pathogenesis of late aneurysms after patch graft aortoplasty for coarctation. J. Thorac. Cardiovasc. Surg., 92: 226-230, 1986.

9

JONAS, R. A. - Coarctation: do we need to resect ductal tissue? Ann. Thorac. Surg., 52: 604-607, 1991.

10 KORFER, R.; MEYER, H.; KLEIKAMP, G.; BIRCKS, W. - Early and late after resection and end-to-end anastomosis of coarctation of the thoracic aorta in early infancy. J. Thorac. Cardiovasc. Surg., 89: 616$622,1985$.

11 LACOUR-GAYET, F.; BRUMIAUX, J.; SERRAF, A.; CHAMBRAM, P.; BLAYSAT, G.; LOSAY, J.; PETIT, J.; KACHANER, J.; PLANCHE, C. - Hypoplastic transverse arch and coarctation in neonates. $J$. Thorac. Cardiovasc. Surg., 100: 808-812, 1990.

MEIER, M. A.; LUCCHESE, F. A.; JAZBIK, W.; NESRALLA, I. A.; MENDONÇA, J. T. - A new technique for aortic coarctation: subclavian flap aortoplasty with preservation of arterial blood flow to the left arm. J. Thorac. Cardiovasc. Surg., 92: 1005$1012,1985$.

13 MENDONÇA, J. T.; CARVALHO, M. R.; COSTA, R. K. - Coarctation of the aorta: a new surgical technique. J. Thorac. Cardiovasc. Surg., 90: 445-447, 1985.

14 MESSMER, B. J.; MINALE, C.; MUHLER, E.; BERMUTH, G. - Surgical correction of coarctation in early infancy: does surgical technique influences the result? Ann. Thorac. Surg., 52: 594-603, 1991.

15 UNGERLEIDER, R. M. - Is there a role for prosthetic patch aortoplasty in the repair of coarctation? Ann. Thorac. Surg., 44: 321-323, 1987.

16 UNGERLEIDER, R. M. \& EBERT, P. A. - Indication and techniques of medline approach to aortic coarctation in infants and children. Ann. Thorac. Surg., 44: 517 $522,1987$.

17 VOSSCHULTE, K. - Surgical correction of coarctation of the aorta by an "isthmus plastic" operation. Thorac., 16: 338-343, 1961.

18 WALDHAUSEN, J. A. \& NAHRWOLD, D. L. - Repair of coarctation of the aorta with a subclavian flap. $J$. Thorac. Cardiovasc. Surg., 51: 523-525, 1966.

19 WILLIAM, W. G.; SHINDO, G.; TRUSLER, G. A.; DISHE, M. R.; OLLEY, P. M. - Results of repair of coarctation of the aorta during infancy. J. Thorac. Cardiovasc. Surg., 79: 603-608, 1980. 